\title{
Epidemiologic Risk Factors for Breast Cancer Related to Menopausal Status in Indonesia
}

\author{
Sadao Suzuki, Joedo Priha rtonot, Yoshiyuki Ohno', Setyawati Budiningsiht, Kenji Wakai·, Santoso Cornainu, \\ Nakako Kubo II, Muchlis Ra mli ${ }^{11}$ Susumu Wata nabe**, Idral Daiwis ${ }^{11}$ Didit Tjindarbumi ${ }^{11}$ Gunawan Tjahjadit,
}

Esti Soetrisno:t:, Endang Sri Roostini:t:

\begin{abstract}
Abstrak
Untuk meneliti faktor risiko kanker payudara yang berkaitan dengan status menopause, dilakukanpenelitian kasus kontrol pada rumah sakit di Indonesia. Pada 300 kasus dan 600 kontrol yang berpasangan menurul umur dan keadaan sosial ekonomi dilakukan wawancara. Kasus dan kontrol dikelompokkan berdasarkan status menopausenya dan dianalisis terpisah. Diperoleh temuan-temuan bermakna berikut ini.Di antara penderita kanker payudara pra menopause ditemukan peningkatan risikopada yang mengalami trauma payudara (RR: 2,62; 95\% JK: 1,09-6,31), menggunakan kontrasepsi oral (4,96; 1,51-16,24), mengkonsumsi susu (1,81; 1,01-3,35 konsumsi tiap hari versus nir konsumsi), makan buah sega $(2,42 ; 1,16-5,05$, 3-4x per minggu versus kurang dari seka li per minggu). Penurunan risiko ditemukan pada wanita pengkonsumsi sayuran tiap hari (0,34;0,15-0,77, konsumsi tiap hari versus tidak tiap hari). Di antara penderita kanker payuda ra pasca menopause ditemukan peni ngkatan risiko pada wanita dengan usia menard1e 15 tahun atau lebih $(2,25 \cdot 1,35-3,76)$, haid teratur setelah usia 30 tahun $(4,61-\cdot 2,45-8,67)$, konsumsi susu $(5,84 ;$ 2,92-11,66, konsumsi tiap hari versus nir konsumsi). Penurunan risiko ditemukan pada wanita yang cerai atau janda $(0,33 ; 0,18-0,58)$, jumlah melahirkan hidup atau bayi yang disusuinya tinggi (0,32; 0,13-0,76), menyusui 6 atau lebih bayi versus tidak menyusui).
\end{abstract}

\begin{abstract}
To clarify the risk factors of breast cancer in premenopausal and postmenopausal women, a hospital-based case-control study was conducted in Indonesia. Three hundred inciden t cases were inlerviewed and 600 controls were selected, matching for age and socioeconomic class. Cases and controls were divided according to their menopausal status and analyzed separately. Following significant findi ngs were revea led. For premenopausal breast cancer, an increased risk was detected in women with breast trauma (adjus ted $R R$ : 2.62; 95\% Cl: 1.09-6.31), oral contraceptive u e (4.96,-1.51-16.24), milk consumption (1.81; 1.01-3.35, daily intake vs no intake), fresh frui ts intake (2-42; 1.16-5.05, 3-4 times/week vs less than once/week intake). A decreased risk was detected in women with cooked-vegetable intake (0.34; 0.15-0.77, daily intake vs not daily intake). For postmenopausal breast cancer, an increased risk wasfound in women having menarche at the age of 15 years or over $(2.25 ; 1.35-3.76)$, regular menstruation after thirty years old (4.61; 2.45-8 .67), milk consumption (5.84; 2.92-11.66, daily intake vs no intake), and a decreased risk in women who were divorced or widowed (0.33; 0.18-0.58), and whose number of live birth or breast-fed children was high $(0.32 ; 0.13-0.76$, six or more breast-fed children vs no breast-fed child).
\end{abstract}

Keywords :case-control study, premenopausal women, post menopausal women, breast cancer, risk factors

Deparlme11/ of Preventive Medicine, School of Medicine Nagoya U11iversily, Nag oya 466, Japan

t Deparlme11t of Comm1111ity Medici11e, Fac11/ty of Medicine, U11iversity oflndonesia, Jakarta 10320,/ndo11esia

;f: Department of Anatomic Palhology, Fawlty of Medicine, University of Ind onesia, Jaka rta 10430, Indona;ia

§ Research Center, Faculty of Medicine, University of Indonesia, Jakarta 10430,Jndon.esia

IIDepartment of Public Health, Medical School, Nagoya City University, Nag oya 467, Japan

${ }^{11}$ Department ofS11rgery, Family ofMedicine, University of Indonesia, lakarta 10430, Jndo11esia

Departme11/ of Surgery, Cancer Institute Hospital, To/..yo 170, Japan
Risk factors of breast cancer (BC) have been widely discussed, and different etiologic factors have been suggested related to menopausal status. ${ }^{16}$ BC risk has been reported to be negatively associated with the number of pregnancies. This protective effect may apply only to women whose BC was diagnosed at 50 years or older.7 A few studies suggest that parity may be associated with an increased risk of $\mathrm{BC}$ in young women. ${ }^{8}-9$

In postmenopausal women, body weight and other indicators of weight (weightlhe\%ht ratio) were positively associated with the risk, whereas in premenopausal women, a negative association has been reported between $\mathrm{BC}$ risk and weight. ${ }^{11} \mathrm{BC}$ risk in- 
creased in first-degree rela tives of premenopausal BC patient, but not in relatives of postmenopausal BC patients. ${ }^{3}$ Lactation showed protective effect against premenopausal BC alone. ${ }^{36}$ The studies exemplified above strongly support the idea that premenopausal women differ from postmenopausal women concerning $\mathrm{BC}$ risk factors.

In view of the above differences, we have analyzed the risk factors in premenopausal and post menopausal women separately in this paper.

\section{METHODS}

Methodological details of the case control study design and data collection were already described in the preceeding paper. In this paper, cases and controls were grouped according to their menopausal status and were analysed separately. Relative risk (RR) with its 95\% confidence interval (CI), computed as the exposure-odds ratio, was used as a measure of the associations between potentia 1 risk factors and BC. Trends were evaluated by the Mantel extension test. ${ }^{12}$ To account for age, socioeconomic class, residence and other va riables potentia lly confounded, unconditional logistic regression analysis ${ }^{13}$ was performed.

\section{RESULTS}

Out of 300 histologically diagnosed BC patients examined from December 1988 to November 1991 at
Cipto Mangunkusumo Central Hospital, University of Indonesia, 135and 163patients were at premenopausal and postmenopausal status respectively. Two patients were at unknown menopausal status. The age distribution of cases and controls is shown in Table 1. Postmenopausal cases were significantly you nger than their controls (54.9 vs. 57.4 years old), whereas premenopausal cases were not significantly so (37.5 vs. 38.2 years old). Table 2 shows the mea $n$ and standard deviation (SD) of height, weight and obesity indices as BMI and waist / hip ratio. In postmenopausal women, controls were significantly taller by $1.2 \mathrm{~cm}$, and slightly smaller in waist $/$ hip ratio than cases. Excluding these differences, no other significant casecontrol differences were found in both premenopausal and post-menopausal study subjects.

Table 3 shows the RRs of breast cancer in premenopausal women obtained by univariate analysis. Breast trauma and the use of oral contraceptives significantly increased the risk. History of malignant neoplasm among relatives (mother, aunts or sisters) increased the risk by $91 \%$, though the increase was not significa nt. The risk increased with increasing milkintake and amount of fresh fruits consumed; significant linear trends were found (chi-squa re for trend $=4.83$, $\mathrm{P}<0.05$ and $4.68, \mathrm{P}<0.05$ respectively). Daily inta ke of cooked vegetables significantly decreased the risk.

Ta ble 1. Age Distribution of Cases and Controls in Premenopa usa l and Postmenopa usal group

\begin{tabular}{|c|c|c|c|c|c|c|c|c|}
\hline \multirow{3}{*}{ Age } & \multicolumn{4}{|c|}{ Premenopausa l group } & \multicolumn{4}{|c|}{ Postmenopausal group } \\
\hline & \multicolumn{2}{|l|}{ Cases } & \multicolumn{2}{|l|}{ Controls } & \multicolumn{2}{|l|}{ Cases } & \multicolumn{2}{|l|}{ Controls } \\
\hline & Number & $\%$ & Number & $\%$ & Number & $\%$ & Number & $\%$ \\
\hline $20-29$ & 14 & 10.4 & 30 & $<\mathrm{u}$ & & & & \\
\hline 30-39 & 60 & 44.4 & 163 & 49.5 & 10 & 6.2 & 4 & 1.5 \\
\hline $40-49$ & 59 & 43.7 & 124 & 37.7 & 33 & 20.4 & 31 & 11.4 \\
\hline $50-59$ & 2 & 1.5 & 12 & 3.7 & 52 & 32.1 & 115 & 42.4 \\
\hline $60-69$ & & & & & 58 & 35.8 & 107 & 39.5 \\
\hline $70-79$ & & & & & 9 & 5.6 & 14 & 5.2 \\
\hline Totals & 135 & 100.0 & 329 & 100.0 & 162 & 100.0 & 271 & 100.0 \\
\hline Mean age \pm S.D. & 37.5 & fi...4 & 38.2 & $6.9 \mathrm{~N} \mathrm{~S}$ & 54.9 & 9.4 & 57.4 & 7.6 \\
\hline
\end{tabular}


Table 2. Mean Val ues of Height, Weight and Obesity Indicators

\begin{tabular}{|c|c|c|c|c|c|c|c|c|c|}
\hline & \multicolumn{4}{|c|}{ Premenopausa l group } & \multicolumn{5}{|c|}{ Postmenopa usal group } \\
\hline & \multirow[t]{2}{*}{ Number } & \multicolumn{3}{|c|}{ Mea ns :1: S.D. } & \multirow[t]{2}{*}{ Number } & \multicolumn{3}{|c|}{ Mea ns :1: S.D. } & \\
\hline \multicolumn{8}{|l|}{ Height $\quad(\mathrm{cm})$} & & \\
\hline Cases & 133 & 153.4 & :I: 5.1 & NS & 161 & 152.3 & : : & 5.2 & \\
\hline Controls & 324 & 153.4 & :I: 5.0 & & 269 & 153.5 & : : & 5.4 & \\
\hline \multicolumn{10}{|l|}{ Weight (kg) } \\
\hline Cases & 134 & 49.6 & :I: 8.8 & NS & 161 & 50.8 & : : & 10.6 & NS \\
\hline Controls & 325 & 50.0 & :I: 6.7 & & 270 & 51.5 & & 8.2 & \\
\hline \multicolumn{10}{|c|}{ Maxi mum weight (kg) } \\
\hline Otses & 94 & 52.8 & :I: 7.8 & NS & 119 & 54.8 & & 10.9 & NS \\
\hline Controls & 223 & 52.5 & :I: 7.9 & & 211 & 55.5 & :I: & 8.6 & \\
\hline \multicolumn{10}{|l|}{ Waist (cm) } \\
\hline Cases & 133 & 70.0 & :I: 7.9 & NS & 160 & 74.2 & & 11.5 & NS \\
\hline C. ontrols & 323 & 69.8 & :I: 7.5 & & 269 & 72.9 & :I: & 9.2 & \\
\hline \multicolumn{10}{|l|}{ Hip (cm) } \\
\hline Cases & 132 & 91.3 & :1: 9.7 & NS & 161 & 94.1 & :: & 13.3 & NS \\
\hline O.rntrols & 323 & 91.5 & :I: 9.1 & & 270 & 95.0 & : : & 10.6 & \\
\hline \multicolumn{10}{|l|}{ BMI $(\mathrm{kg} / \mathrm{m} / \mathrm{m})$} \\
\hline Cases & 133 & 21.0 & : : 3.3 & NS & 160 & 21.9 & :l: & 4.4 & NS \\
\hline nrntrols & 325 & 21.2 & :I: 2.7 & & 269 & 21.9 & : : & 3.2 & \\
\hline \multicolumn{10}{|c|}{ Waist / hip ratio } \\
\hline Otses & 132 & 0.77 & :I: 0.09 & NS & 160 & 0.79 & : : & 0.09 & \\
\hline Controls & 321 & 0.77 & : 0.09 & & 269 & 0.77 & : : & 0.09 & \\
\hline
\end{tabular}

NS = not significa nt, '0.05 2: $\mathrm{p}>0.01$,

Table 3. Distri bution of Risk Factors in Premenopa usal Group

\begin{tabular}{|c|c|c|c|c|c|}
\hline Factors & Cases & Controls & R.R. & 95\% C.l. & Trend \\
\hline \multicolumn{6}{|l|}{ Breast tra uma } \\
\hline Never & 120 & 300 & 1.00 & \multirow{2}{*}{\multicolumn{2}{|c|}{$\begin{array}{l}\text { Reference } \\
(1.37-6.07)\end{array}$}} \\
\hline Ever & 15 & 13 & 2.88 & & \\
\hline \multicolumn{6}{|l|}{ Pill use } \\
\hline No & 126 & 324 & 1.00 & \multirow{2}{*}{\multicolumn{2}{|c|}{$\begin{array}{l}\text { Reference } \\
(1.67-12.84)\end{array}$}} \\
\hline Yes & 9 & 5 & 4.63 & & \\
\hline \multicolumn{6}{|l|}{ Maligna ncy a mong rela tives } \\
\hline No & 107 & 290 & 1.00 & \multirow{2}{*}{\multicolumn{2}{|c|}{$\begin{array}{l}\text { Reference } \\
(1.03-3.54)\end{array}$}} \\
\hline Yes & 19 & 27 & 1.91 & & \\
\hline \multicolumn{6}{|l|}{ Milk } \\
\hline Never & 38 & 98 & 1.00 & \multirow{5}{*}{$\begin{array}{l}\text { Reference } \\
(0.43-1.37) \\
(0.49-1.71) \\
(0.45-2.07) \\
(1.06-3.23)\end{array}$} & \multirow[t]{5}{*}{4.83 * } \\
\hline Less than once/week & 26 & 87 & 0.77 & & \\
\hline Once-twice/week & 21 & 59 & 0.92 & & \\
\hline 3-4 ti mes/week & 12 & 32 & 0.97 & & \\
\hline Daily & 38 & 53 & 1.85 & & \\
\hline \multicolumn{6}{|l|}{ Fresh fruits } \\
\hline Never - less tha n once /week & 23 & 86 & 1.00 & \multirow{4}{*}{$\begin{array}{l}\text { Reference } \\
(0.48-2.23) \\
(1.36--:-5.05) \\
(1.00-2.91)\end{array}$} & \multirow[t]{4}{*}{4.68} \\
\hline Once-twice/week & 13 & 47 & 1.03 & & \\
\hline 3.4 ti mes/week & 28 & 40 & 2.62 & & \\
\hline Daily & 71 & 156 & 1.70 & & \\
\hline \multicolumn{6}{|l|}{ Cooked vegetable } \\
\hline Not daily & 18 & 14 & 1.00 & \multirow{2}{*}{\multicolumn{2}{|c|}{$\begin{array}{l}\text { Reference } \\
(0.14-0.58)\end{array}$}} \\
\hline Daily & 117 & 315 & 0.29 & & \\
\hline
\end{tabular}

0.05 2: $\mathrm{P}>\mathrm{O} . \mathrm{Dl}$ 
Table 4. Distribution of Risk Factors in Postmenopausal Group

\begin{tabular}{|c|c|c|c|c|c|}
\hline Factors & Otses & Controls & R.R. & 95\% C.I. & Trend \\
\hline \multicolumn{6}{|l|}{ Marital status } \\
\hline Married & 86 & 95 & 1.00 & Reference & \\
\hline Separated or widowed & $68^{\prime}$ & 173 & 0.43 & $(0.29-0.65)$ & \\
\hline Unmarried & 9 & 3 & 3.31 & $(0.93-11.84)$ & \\
\hline \multicolumn{6}{|l|}{ Height (cm) } \\
\hline$<1-\mathrm{SO}$ & 36 & 50 & 1.00 & Reference & 4.30 \\
\hline $150-159$ & 113 & 177 & 0.89 & $(0.54-1.45)$ & \\
\hline i!: 160 & 14 & 44 & 0.44 & $(0.21-0.92)$ & \\
\hline \multicolumn{6}{|l|}{ Waist / hip ra tio } \\
\hline$<0.70$ & 28 & 65 & 1.00 & Reference & 5.53 \\
\hline $0.70-0.79$ & 57 & 101 & 1.31 & $(0.76-2.27)$ & \\
\hline $0.80-0.89$ & 53 & 84 & 1.46 & $(0.84-2.56)$ & \\
\hline i!: 0.90 & 22 & 19 & 2.69 & $(1.27-5.67)$ & \\
\hline \multicolumn{6}{|l|}{ Mena rche } \\
\hline$<15$ years old & 71 & 92 & 1.00 & Reference & $18.31 \mathrm{t}$ \\
\hline i!: 15 years old & 175 & 95 & 2.36 & $(1.60-3.50)$ & \\
\hline \multicolumn{6}{|c|}{ Regularity of menstruation over 30 years } \\
\hline Irregula r & 33 & 105 & 1.00 & Reference & $18.51 \mathrm{t}$ \\
\hline Regula r & 130 & 165 & 2.51 & $(1.60-3.92)$ & \\
\hline \multicolumn{6}{|l|}{ Menopa usa l process } \\
\hline Natural & 144 & 263 & 1.00 & Reference & \\
\hline Induced & 19 & 8 & 4.34 & $(1.97-9.57)$ & \\
\hline \multicolumn{6}{|l|}{ Num ber of live birth } \\
\hline None & 25 & 22 & 1.00 & Reference & $26.07 \mathrm{t}$ \\
\hline 1,2 & 44 & 35 & 1.11 & $(0.54-2.28)$ & \\
\hline $3-5$ & 55 & 150 & 0.32 & $(0.17-0.61)$ & \\
\hline i!: 6 & 36 & 62 & 0.52 & $(0.25-1.03)$ & \\
\hline \multicolumn{6}{|l|}{ Num ber of lactation } \\
\hline None & 29 & 27 & 1.00 & Reference & $28.83 t$ \\
\hline 1,2 & 45 & 33 & 1.27 & $(0.64-2.53)$ & \\
\hline $3-5$ & 56 & 153 & 0.34 & $(0.19-0.62)$ & \\
\hline 2: 6 & 31 & 57 & 0.51 & $(0.26-1.00)$ & \\
\hline \multicolumn{6}{|l|}{ Smoking habit } \\
\hline Nonsmoker & 148 & 259 & 1.00 & Reference & \\
\hline Current ex-smoker & 15 & 12 & 2.19 & $(1.00-4.72)$ & \\
\hline \multicolumn{6}{|l|}{ Milk } \\
\hline Never & 54 & 138 & 1.00 & Reference & $32.20 \mathrm{t}$ \\
\hline Less than once / week & 24 & 63 & 0.97 & $(0.55-1.71)$ & \\
\hline Once-twice / week & 20 & 27 & 1.89 & $(0.99-3.63)$ & \\
\hline 3-4 ti me, week & 15 & 9 & 4.26 & $(1.85-9.81)$ & \\
\hline Almost daily & 50 & 33 & 3.87 & $(2.29-6.55)$ & \\
\hline
\end{tabular}

0.05 2: $P>0.01$, O.Ql 2: $P>0.001$, to.001 2: $P>0.0001$ 
Table 4 summa rizes the ris $\mathrm{k}$ facto rs for postmenopausal women detected by univa riate analysis. Compared to married women, separated or widowed women were at a smaller risk, but umnarried women were at a greater risk. When the marital status was dichotomized into unmarried and ever ma rried, then unma rried women showed a RR of 5.22 (95\% Cl: 1.58-17.21). Height was inversely associated with BC $(P<0.05)$, while the larger the waist $I$ hip ratio, the higher the risk $(P<0.05)$. When compared to those with menarche at less than 15 years old, those with menarche at 15 yea rs old or more had a higher RR. Regula $r$ me nst ru a ti o n a fter 30 yea rs ol d a nd ind uced menopause increased the risk. Since the number of live birth and that of lactation showed very similar distribution, they showed similar RRs. When compared with nulliparous women, those with one or two births had a RR of 1.11(0.54-2.28), 3-5 births $0.32(0.17-0.61)$ and 6 or more births $0.52(0.25-1.03)$; chi-square for trend being $26.07(\mathrm{P}<0.001)$. Similarly when compared to women with no breast-fed child, those with one or two breast-fed children had a RR of 1.27 (0.64-2.53), 3-5 children 0.34 (0.19-0.64) and 6 or more children 0.51 (0.26-1.00); chi -sq ua re for trend bei ng 28.83 $(\mathrm{P}<0.001)$. Compared to nonsmokers, current or exsmoker experienced a higher risk. Postmenopausal women who drunk milk less than once per week had a RR of 0.97 (0.55-1.71), once to twice per week 1.89 (0.99-3.63), 3-4 times per week 4.26 (1.85-9.81), almost daily 3.87 (2.29-6.55), when compared to non$\mathrm{d}$ rinker of milk; linea $\mathrm{r}$ trend bei ng signi ficant $(\mathrm{P}<0.001)$.

After being adjusted for age, socioeconomic class, residence and other variables potentially confounded, by u nconditiona l logistic regression analysis, the results (Table 5,6) were quite similar to those before adjustment. Association between BC with maligna ncy among relatives (premenopausal), height, waist $I$ hip ratio, unmarried status, smoking habit and menopausal process (postmenopausa l) became insignificant or marginally significant. The number of live births was not included in the logistic regression a nalysis, since its distribution was very similar to that of the number of breast-fed children.

Table 5. Adjusted Rela tive Risks of Breast Cancer in Unconditional Logistic Ana lysis in Premenopausal Group

\begin{tabular}{lll}
\hline Factors & R.R. & $95 \%$ C.I. \\
\cline { 2 - 3 } $\begin{array}{l}\text { Breast tra uma } \\
\quad \text { Never }\end{array}$ & 1.00 & Reference \\
$\quad$ Ever & 2.62 & $(1.09-6.31)$ \\
Pill use & & \\
$\quad$ No & 1.00 & Reference \\
$\quad$ Yes & 4.96 & $(1.51-16.24)$ \\
Mal igna ncy among rel atives & & \\
$\quad$ No & 1.00 & Reference \\
$\quad$ Yes & 1.99 & $(0.98-4.02)$ \\
Mil k & & \\
$\quad$ Never & 1.00 & Reference \\
$\quad$ Less tha n once/week & 0.81 & $(0.42-1.54)$ \\
$\quad$ Once-twice/week & 0.99 & $(0.50-1.95)$ \\
3-4 ti mes/week & 0.97 & $(0.42-2.23)$ \\
$\quad$ Daily & 1.81 & $(1.01-3.35)$ \\
Fresh fruits & & \\
$\quad$ Never - less than once/week & 1.00 & Reference \\
Once-twice/ week & 0.70 & $(0.29-1.67)$ \\
3-4 ti mes/week & 2.42 & $(1.16-5.05)$ \\
Daily & 1.40 & $(0.74-2.66)$ \\
O:ioked vegeta ble & & \\
Not daily & 1.00 & Reference \\
Daily & 0.34 & $(0.15-0.77)$ \\
\hline
\end{tabular}


Table 6. Adjusted Rela tive Risks of Breast Cancer in Unconditional Logistic Analysis in Postmenopa usal Group

\begin{tabular}{|c|c|c|}
\hline Factors & R.R. & 95\% C.I. \\
\hline \multicolumn{3}{|l|}{ Marital status } \\
\hline Married & 1.00 & Reference \\
\hline Separated or widowed & 0.33 & $(0.18-0.58)$ \\
\hline Unmarried & 2.71 & $(0.31-23.63)$ \\
\hline \multicolumn{3}{|l|}{ Height (cm) } \\
\hline$<150$ & 1.00 & Reference \\
\hline $150-159$ & 0.74 & $\left(\begin{array}{ll}0.40 & 1.36\end{array}\right)$ \\
\hline 160 & 0.43 & $(0.17-1.13)$ \\
\hline \multicolumn{3}{|l|}{ Waist / hip ratio } \\
\hline$<0.70$ & 1.00 & Reference \\
\hline $0.70-0.79$ & 0.99 & $\left(\begin{array}{ll}0.48 & 2.04\end{array}\right)$ \\
\hline $0.80-0.89$ & 0.44 & $(0.20$ \\
\hline 0.90 & 0.81 & $(0.31$ \\
\hline \multicolumn{3}{|l|}{ Menarche } \\
\hline$<15$ years old & 1.00 & \multirow{2}{*}{$\begin{array}{l}\text { Reference } \\
(1.35-3.76)\end{array}$} \\
\hline 15 years old & 2.25 & \\
\hline \multicolumn{3}{|c|}{ Regularity of menstruation over 30 years } \\
\hline Irregular & 1.00 & \multirow{2}{*}{$\begin{array}{l}\text { Reference } \\
(2.45-8.67)\end{array}$} \\
\hline Regular & 4.61 & \\
\hline \multicolumn{3}{|l|}{ Menopa usal process } \\
\hline Natural & 1.00 & \multirow{2}{*}{$\begin{array}{l}\text { Reference } \\
(0.84-7.74)\end{array}$} \\
\hline Induced & 2.54 & \\
\hline \multicolumn{3}{|l|}{ Num ber of lactation } \\
\hline None & 1.00 & Reference \\
\hline $1-2$ & 1.15 & $\left(\begin{array}{ll}0.48 & 2.72\end{array}\right)$ \\
\hline $3-5$ & 0.29 & $(0.13$ \\
\hline 6 & 0.32 & $(0.13$ \\
\hline \multicolumn{3}{|l|}{ Smoking habit } \\
\hline Nonsmoker & 1.00 & \multirow{2}{*}{$\begin{array}{l}\text { Reference } \\
(0.76-7.09)\end{array}$} \\
\hline C'.urrent/ex-smoker & 2.33 & \\
\hline \multicolumn{3}{|l|}{ Milk } \\
\hline Never & 1.00 & Reference \\
\hline Less than once / week & 1.13 & $(0.55-2.33)$ \\
\hline Once-twice / week & 2.26 & $(0.97-5.29)$ \\
\hline 3-4 ti mes / week & 7.96 & $\left(\begin{array}{ll}2.63 & -24.13\end{array}\right)$ \\
\hline Almost daily & 5.84 & (2.92 -11.66) \\
\hline
\end{tabular}

\section{DISCUSSION}

In the present study, we found the association between premenopausal BC and breast trauma, oral contraceptive use, high intake of milk and fresh fruits, and low intake of cooked vegetable.

Benign breast diseases have been reported as BC risk factor, ${ }^{11,14}$ but there have been no reports on the relationship between breast trauma and BC risk. Therefore, studies are required to confirm such association.

Many reports noted that the use of oral contraceptives did not affect BC risk, ${ }^{15}{ }_{-}^{17}$ while our study revealed the contrary. This might be due to the small number of pill users in our series, thus our finding might possibly be obtained by chance. In the present study, mil k intake had positive association with BC risk and significa nt linea $r$ trend was observed among both premenopausa $\mathrm{l}$ and postmenopausa $\mathrm{l}$ group. Milk might representedie8aR' fat which has long been in-

- $\quad$ Protective effect of cooked vegetables might be due to the vitamins, such as vitamin $A$ and beta-carotene, though inconsistent resu Its ${ }^{20-22}$ were reporte ${ }^{\mathrm{d}}$. The assoclation between fresh fruits intake and premenopausal BC might be related to the level of total calory intake. ${ }^{23}$

Associations between postmenopausal BC risk with ma rita 1 status, age at menarche, regula r menstruation 
after thirty years old, induced menopause, number of live birth or breast-fed child ren and milk consumption were noted in this study.

Our study showed that unmarried women had 5.2 times higher risk than married women. Unmarried women hâd 2 a higher risk, although some reports did not prove it. . The reason why separated or widowed women were at a lower risk of postmenopausal BC was unclear.

Menarche at older age was associated with postmenopausal BC in this study, but this is not a common finding. ${ }^{11} 25$ Regula $r$ menstruation before menopause is known to be a risk factor of $\mathrm{BC},{ }^{26} \cdot 27$ and our finding support this. Induced menopause was found to be a risk factor for postmenopa usal BC by our univa riate analysis, but not by logistic regression analysis. No risk difference was detected between natural and induced menopause. ${ }^{28}$

An inverse linear trend between postmenopausal BC and the number of live birth $I$ breast fed children remained significant after adjusting for age and other

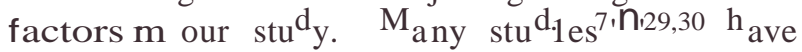
reported that an increasing number of pregnancy or delivery has an independent protective effect. This protective effect was found more consistently in older or postmenopausal women. A few studies ${ }^{8} \cdot{ }^{9}$ suggest that parity ma be associa ted with an increased risk for $\mathrm{BC}$ in young women. These are in good agreement with our findings.

Postmenopausal women who had smoking experience had approximately a two-fold BC risk in our study, however, this was not significant by logistic regression analysis. Most reports suggested no clear association between $B C$ and ciga rette $s m o k_{m}^{\prime} h_{a} b_{i t s .}^{\prime}{ }^{31} 32$

\section{CONCLUSION}

Our study disclosed some different BC risk factors in premenopa usa $l$ and postmenopa usa $l$ women. We found more risk factors and stronger associations in postmenopausal than in premenopausal women. Milk inta ke was a common risk factor, which might represent fat intake.

\section{Acknowledgments}

The authors are grateful to the nurses and the public health nurses for excellent care and data collection from breast cancer cases and controls. This work was supported by the Ministry of Education and Culture, Japan, Grants No. 01042007 and 04042013; and was partly supported by the Indonesian Cancer Foundation. This collaborative study was a part of Special Cancer Research Project in Monbusho International Scientific Research Program, with the approval of the Dean, Faculty of Medicine, University of Indonesia, No. 4383/PT02.H4.FK/E/88.

\section{REFERENCES}

l. Oemmesen J . Carcinoma of the breast: Resul ts from statistical research. Br J Radiol 1948;21:583-90.

2. DeWaard F, Baanders-Van Halewijn EA, Huizi'ngs J. The bi modal age distribution of patients with mammary carcinoma. Ca ncer 1964;17:141-52.

3. Anderson DE. A genetic study of human breast cancer. J NCI 1972;48:1029-34.

4. Craig T, Camstock GW, Geiser GB. Epidemiologic comparison of breast cancer patients with early and late onset of ma 1 igna ncy a nd genera 1 popula tion controls. JNCI 1974;53:1577-81.

5. Byere T, Graham S, Rzepka, Marshall J. Lactation and breast cancer. Am J Epi demiol 1985;121:664-74.

6. McTiernan A, Thomas DB. Evidence for a protective effect of lactation on risk of breast cancer in young women. Am J Epidemiol 1986;124:353-8.

7. Leon DA. A prospective study of the independent effects of pari ty and age at first birth on breast cancer incidence in Engl and and Wal es. Int J Ca ncer 1989;42:986-91.

8. Patha k DR, Speizer FE, Willet WC, Rosner B, Lipnick RJ. Pa rity and breast cancer risk: possi ble effect on age at diagnosis. Int J Cancer 1986;37:21-5

9. Negri E, La Vecchia C, Bruzzi P,Dardanoni G, Deearli A, Palli D, et al. Risk factors for breast cancer: period resul ts from three Italian case-control studies. Am J Epidemiol 1988;128:1207-15

10. Swanson CA, Brinton LA, Taylor PR, Licitra LM, Ziegler RG, Schairer C. Body size and breast cancer risk assessed in women participating in the breast cancer detection demonstra ti on project. Am J Epidemiol 1989;130:1133-41.

11. Yua n JM, Yu MC, Ross RK, Gao YT, Henderson BE. Risk factors for breast cancer in Chinese women i n Shanghai. Cancer Res 1988;48:1949-53.

12. Breslow NE, Day NE.The analysis of case-control study [n: Davis W, editor. Statistical methods in cancer research. Vol. I. Lyon: IARC, 1980:146-54.

13. Breslo WNE, Day NE. Uncondtional logistic regression for large strata. In: Davis W, editor. Statistica l methods in cancer research, Vol. I. Lyon: IARC, 1980:192-246.

14. Siski nd V, Schofield F, Rice D, Bain C. Breast cancer and breast feeding: results from an Australian case-control study. Am J Epidemiol 1989;130:229-36.

15. Miller DR, Rosenberg L, Kaufman DW, Stolley P, Warshauer ME, Shapiro S. Breast cancer before age 45 and oral contra cepti ve use: new fi nd i ngs. Am J Epidem i ol 1989;129:269-80.

16.Stanford JL, Bri nton LA, Hoover RN. Oral contraceptive and breast cancer: results from an expa nded case-control study. Br J Cancer 1989;60:275-81. 
17. UK National Case-Omtrol Study Group. Oral contraceptive use a nd breast cancer risk i n you ng women. La ncet 1989;1:931-82.

18. Willet WC, Sta mpfer MJ, Col ditz GA, Rosner BA, Henneken SCH, Speizer FE. Dietary fat and the risk of breast cancer. N Engl J Med 1987;316:22-8.

19. Hiroha ta T, Nomura AM, Hankin JH, Kolonel LN, Lee J. An epidemiologic study on the associa tion between diet and breast cancer. JNCI 1987;78:595-600.

20. Ka tsouyan ni K, Willet WC, Tri chopoulos D, Boyle P, Trichopoulo A, Vasi laros S, et al. Risk of breast cancer among Greek women in relation to nutrient intake. Cancer 1988;61:181-5

21. Rohan TE, McMichael AJ, Bagharst PA. A popula tionbased case-control study of diet and breast ca ncer i n Austral ia. Am J Epidemiol 1988;128:478-89.

22. Russel MJ, Thomas BS, Bui brook RD. A prospective study of the rel ationship between serum vitami ns $\mathrm{A}$ and $\mathrm{E}$ and risk of breast cancer. Br J Cancer 1988;57:213-15.

23. lscovich JM, lscovich RB, Howe G, Shiboski S, Kaldor JM. A case-control study of diet and breast cancer in Argenti na. Int J Cancer 1989;44:770-6.

24. McTiema n A, Thomas DB. Evi dence for a protective effect of lactati on on risk of breast ca ncer in young woma n. Am J Epi demiol 1986;124:353-8.

25. Hsieh CC, Trichopoulos D, Katsouya nni K, Yuasa S. Age at mena rche, age at menopa use, height and obesity as risk factors for breast cancer: associa tions and interactions in an i nternationa l case-control study. Int J Cancer 1990;46:796800.

26. Olsson H, Landin-olsscn M, Gull berg B. Retrospective assessment of menstrual cycle length in patients wi th breast cancer, in patients with benign breast disease, and women without breast disease. JNCI 1983;70:17-20.

27. Henderson BE, Ross RK, Judo HL, Krailo MD, Pike MC. Do regular ovulatory cycles increase breast cancer risk? Cancer 1985;56:1206-8.

28. Paffeinbarger Jr. RS, Kamper! JB, Chang HG. Characteristics that predict risk of breast cancer before and after the menopa use. Am J Epidemiol 1980;112:258-68.

29. Ewertz M, Duffy SW. Risk of breast cancer in relation to reproductive factors in Denmark. Br J Cancer 1988;58:99104.

30. Bouchardy C, Le MG, Hill C. Risk factors for breast cancer according to age at diagnosis in French case-control study. J Clin Epidemiol 1990;43:267-75.

31. Meara J, McPherson K, Roberts M, Jones L, Vessey M. Alcohol, cigarette smoking and breast cancer. Br J Cancer 1989;60:70-3.

32.N, Triaspolitica. "Kanker Payudara: Informasi, Penyebab, Gejala, Stadium Dan Pengobatan." Mau Nanya Dong Dok. N.p, 28 June 2017. Web. 30 June 2017. $<$ https://nanyadongdok.blogspot.com/2017/06/kanker-payudarainformasi-penyebab.html>. 